\title{
Diagnóstico prenatal de 10 quistes de ovario fetal: manejo posnatal
}

\author{
Raquel Moreno P. ${ }^{1}$, Ricardo Savirón C. ${ }^{1}$, Carolina Corona B. ${ }^{2}$, Diego Lerma P. ${ }^{1}$, \\ Tanit Corbacho G. ${ }^{1}$ \\ 1 Servicio de Obstetricia, Hospital Universitario Miguel Servet. Zaragoza, España. \\ 2 Servicio de Cirugía Pediátrica, Hospital Gregorio Marañón. Madrid, España.
}

\section{RESUMEN}

Antecedentes: Los quistes ováricos fetales son la primera causa de quiste intraabdominal en fetos femeninos. Método: Se recogieron datos maternos, del parto, características del quiste y el manejo. Período de estudio: año 2006 y primer trimestre de 2012. Resultados: Se diagnosticaron 10 casos, todos ellos durante el tercer trimestre. Más frecuentemente unilaterales y de localización izquierda. En 9 de los casos el parto fue eutócico y a término. Uno concluyó en cesárea por fallo de inducción, con pesos fetales y Apgar normales. Sólo uno requirió cirugía posparto, el resto desapareció o redujeron su tamaño posteriormente. Conclusiones: La ecografía del tercer trimestre es fundamental en el diagnóstico y seguimiento de los quistes ováricos fetales, los que suelen tener localización unilateral izquierda. Los quistes ováricos de menor tamaño $(<5$ $\mathrm{cm}$ ), tienen un mejor pronóstico y la mayoría se resuelven espontáneamente o no aumentan su tamaño. Se recomienda una actitud conservadora y seguimiento posnatal de los fetos. Aquellos quistes que superan los $5 \mathrm{~cm}$ y no disminuyen de tamaño pueden precisar tratamiento quirúrgico posparto.

\section{PALABRAS CLAVE: Quiste ovárico fetal, manejo, ultrasonido}

\section{SUMMARY}

Background: Fetal ovarian cysts are the main cause of abdominal cysts in female fetuses. Methods: Were collected maternal data, delivery, cyst characteristics and management. Study period: 2006 and first quarter of 2012. Results: 10 cases were diagnosed, all of them in the third trimester. The majority had unilateral left location. In 9 cases the birth was euthocic and one case of cesarean because of failure of induction. All of them with normal Apgar test and weights. One case needed postnatal surgery. The rest of the ovarian cyst solved spontaneously or reduced their sizes later. Conclusions: Ultrasound of the third trimester is critical in the diagnosis and management of fetal ovarian cysts, which are usually left unilateral location. Ovarian cysts $<5 \mathrm{~cm}$, have a better prognosis and most resolve spontaneously or not increase in size. We recommend a conservative approach and postnatal ultrasound monitoring. Those cysts which exceed $5 \mathrm{~cm}$ and not shrink postpartum may require surgical treatment.

KEY WORDS: Fetal ovarian cyst, management, ultrasound 


\section{INTRODUCCIÓN}

Los quistes fetales de ovario son la primera causa de formación quística intraabdominal en feto de sexo femenino, y la segunda causa de tumoración abdominal detrás de las de origen urológico, sin tener en cuenta el sexo fetal. Su incidencia en centros de referencia es aproximadamente de 1/2.500 (1), sin embargo por su tamaño la gran mayoría son asintomáticos y no son diagnosticados durante la vida fetal. El diagnóstico suele establecerse en el tercer trimestre, debido a que probablemente tengan origen hormonal (2).

El objetivo de esta comunicación es presentar una serie de casos de quiste ovárico fetal y el manejo perinatal de esta situación clínica.

\section{PACIENTES Y MÉTODO}

Se recogieron todos los casos de fetos femeninos que fueron diagnosticados en nuestro centro de quistes ováricos intraútero, en el período comprendido entre el año 2006 hasta principios del 2012. En total se encontraron 10 casos, con una incidencia de 1/2.580 partos, 2 de los cuales tuvieron lugar en una misma gestante, en gestaciones sucesivas. Una vez seleccionados se recogieron datos referentes a la madre (edad, antecedentes médicos y obstétricos), al quiste (semanas de gestación al diagnóstico, tamaño y aspecto, localización y evolución posparto), y parto (tipo de parto, peso y Apgar del recién nacido).

\section{RESULTADOS}

La edad media materna al diagnóstico fue de 34 años (rango: 29-38 años). En 9 de los 10 casos los partos fueron eutócicos, a término (semanas 3741), con un peso medio de 3.350 gramos (rango: 2.930-3.790 g) y con tamaño del quiste inferior a $5 \mathrm{~cm}$. En un caso se realizó inducción de parto a las 36 semanas por el gran tamaño del quiste (90 $\mathrm{mm})$, y finalmente se indicó una cesárea por fallo de inducción. Todos ellos fueron valorados con una buena puntuación de Apgar (8/9, 9/9 ○ 9/10) y tuvieron un $\mathrm{pH}$ de sangre arterial fetal superior a 7,21 (Tabla I).

Tabla I

RESUMEN DE CASOS

\begin{tabular}{|c|c|c|c|c|c|c|}
\hline Caso & Semanas & $\begin{array}{l}\text { Tamaño/ } \\
\text { localización }\end{array}$ & $\begin{array}{l}\text { Tipo parto } \\
\text { (semanas) }\end{array}$ & $\begin{array}{c}\text { Peso } \\
\text { (gramos) }\end{array}$ & Control posparto & $\begin{array}{l}\text { Control 4-6 } \\
\text { meses }\end{array}$ \\
\hline 1 & 32 & $\begin{array}{l}50 \mathrm{~mm} \\
\text { izquierdo }\end{array}$ & $\begin{array}{l}\text { Eutócico } \\
\text { (39) }\end{array}$ & 3330 & Quiste $60 \mathrm{~mm}$ & Resolución \\
\hline 2 & 34 & $\begin{array}{l}17 \mathrm{~mm} \\
\text { izquierdo }\end{array}$ & $\begin{array}{l}\text { Eutócico } \\
\text { (37) }\end{array}$ & 3280 & Resolución & \\
\hline 3 & 30 & $\begin{array}{c}94 \mathrm{~mm} \\
\text { derecho }\end{array}$ & $\begin{array}{c}\text { Cesárea: } \\
\text { fallo de inducción } \\
(36+2)\end{array}$ & 2960 & $\begin{array}{l}\text { Laparatomía: } \\
\text { Resolución }\end{array}$ & \\
\hline $\begin{array}{l}4 \text { (Recidiva } \\
\text { Caso 3) }\end{array}$ & 32 & $\begin{array}{l}28 \mathrm{~mm} \\
\text { izquierdo }\end{array}$ & $\begin{array}{c}\text { Eutócico } \\
(41)\end{array}$ & 3570 & $\begin{array}{l}2 \text { quistes: } \\
\text { Izquierdo: } 89 \mathrm{~mm} \\
\text { Derecho: } 48 \mathrm{~mm}\end{array}$ & Resolución \\
\hline 5 & 34 & $\begin{array}{l}32 \mathrm{~mm} \\
\text { izquierdo }\end{array}$ & $\begin{array}{l}\text { Eutócico } \\
\text { (39) }\end{array}$ & 3200 & $\begin{array}{c}\text { Disminución de } \\
\text { tamaño (14 mm). }\end{array}$ & Resolución \\
\hline 6 & 34 & $\begin{array}{c}38 \mathrm{~mm} \\
\text { izquierdo }\end{array}$ & $\begin{array}{l}\text { Eutócico } \\
\text { (38) }\end{array}$ & 2930 & Resolución & \\
\hline 7 & 32 & $\begin{array}{l}45 \mathrm{~mm} \\
\text { izquierdo }\end{array}$ & $\begin{array}{l}\text { Eutócico } \\
\text { (39) }\end{array}$ & 3340 & $\begin{array}{l}\text { Disminución de } \\
\text { tamaño (32 mm). }\end{array}$ & Resolución \\
\hline 8 & 39 & $\begin{array}{c}29 \mathrm{~mm} \\
\text { izquierdo }\end{array}$ & $\begin{array}{l}\text { Eutócico } \\
\text { (39) }\end{array}$ & 3190 & Resolución & \\
\hline 9 & 34 & $\begin{array}{l}20 \mathrm{~mm} \\
\text { izquierdo }\end{array}$ & $\begin{array}{l}\text { Ventosa } \\
\text { (39) }\end{array}$ & 3790 & Resolución & \\
\hline 10 & 33 & $\begin{array}{l}29 \mathrm{~mm} \\
\text { izquierdo }\end{array}$ & $\begin{array}{c}\text { Eutócico } \\
\text { (39) }\end{array}$ & 3250 & $\begin{array}{c}\text { Disminución } \\
\text { tamaño }(15 \mathrm{~mm})\end{array}$ & Resolución \\
\hline
\end{tabular}


La detección de los quistes, desde $17 \mathrm{~mm}$ hasta $90 \mathrm{~mm}$, fue mediante la ecografía (Figura 1) realizada en el tercer trimestre de gestación, siendo la localización izquierda la más frecuente. Solamente se diagnosticó un caso de localización derecha y otro caso de bilateralidad tras el nacimiento.

Tras el parto se realizaron controles ecográficos de los quistes en 9 de los 10 recién nacidos, que volvieron a repetirse entre los 4 y 6 meses posparto: en 3 casos los quistes ováricos fetales estaban resueltos en el control inmediato posparto y en otros 3 casos los quistes habían disminuido su tamaño posparto, resolviéndose espontáneamente antes de los 6 meses. Solamente en un caso el quiste fue algo mayor en el control posparto, aunque igualmente se resolvió antes de los 6 meses de vida del recién nacido. En otro caso se descubrió la bilateralidad de los quistes en la ecografía de control posparto, desapareciendo ambos quistes en el control posterior.

El quiste diagnosticado de mayor tamaño requirió cirugía laparotómica en los primeros días de vida del recién nacido por el efecto masa ejercido por el quiste a nivel torácico y por la dificultad respiratoria neonatal, resolviéndose tras la misma (Tabla I).

\section{DISCUSIÓN}

Los quistes fetales son la primera causa de quiste intraabdominal en fetos de sexo femenino y la segunda causa de tumoración abdominal por detrás de las causas de origen urológico. Nuestra incidencia coincide con la publicada en centros de referencia, aproximadamente $1 / 2.500$ partos (1). La hipótesis más aceptada para explicar su origen es la hormonal, como respuesta al estímulo de los estrógenos maternos y a la gonadotropina coriónica humana. Además su incidencia se ha visto relacionada con patologías maternas tales como el hipotoroidismo o la diabetes (2).

La gran mayoría corresponden a quistes foliculares, pero también se puede tratar de quistes tecaluteínicos, teratomas o cistoadenomas, sólo el

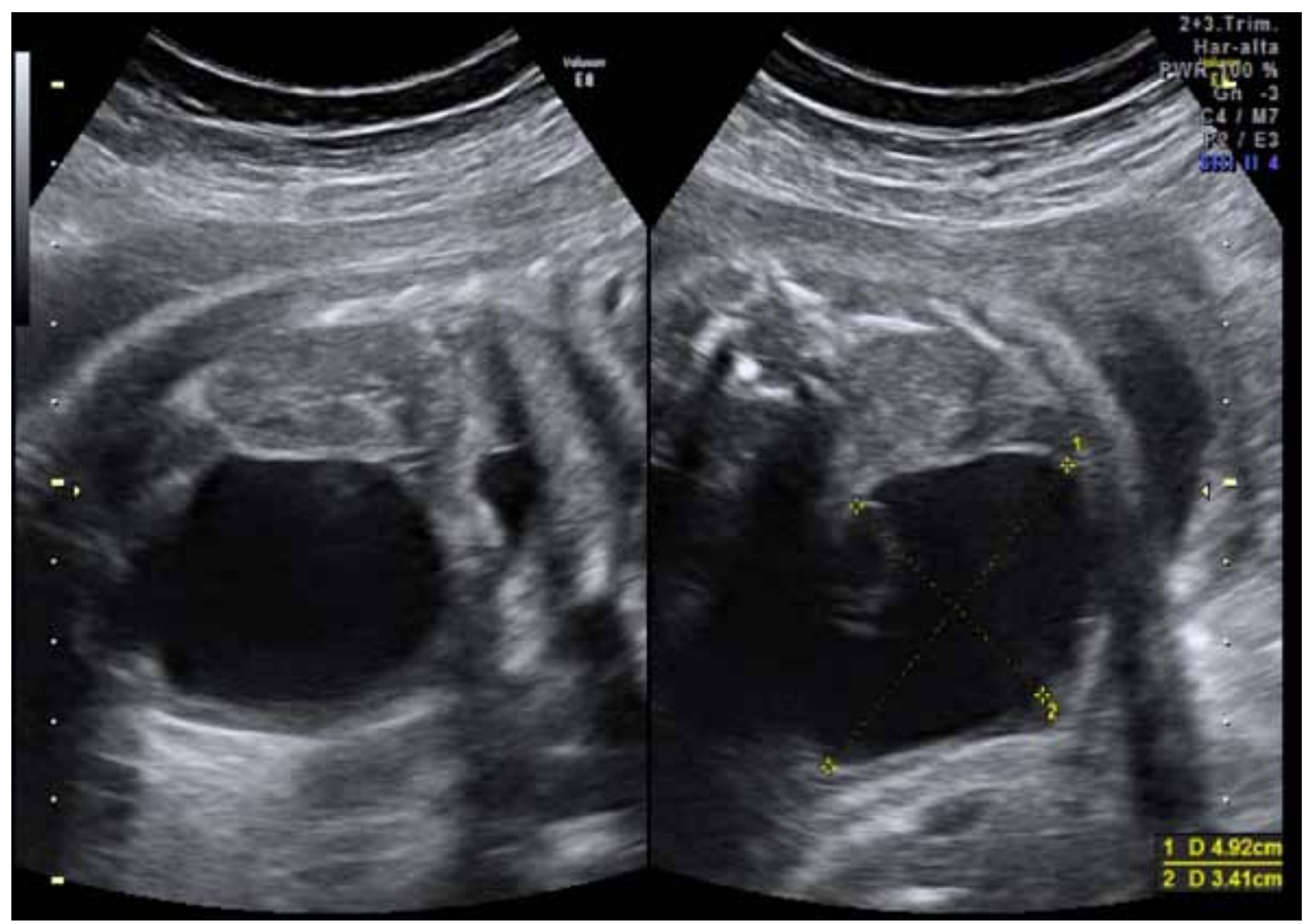

Figura 1. Quiste de ovario fetal (32 semanas): imagen econegativa unilocular en hemiabdomen inferior y lateral, con signo "daugther cyst". 
$10 \%$ corresponden a lesiones orgánicas, raramente malignas (3). En general y si tienen pequeño tamaño, todos se resolverán espontáneamente (4).

Como en nuestros casos, son fundamentalmente unilaterales, frecuentemente izquierdos, no tabicados (5). El diagnóstico habitual en el feto es mediante la ecografía realizada en el tercer trimestre de la gestación (6), donde veremos una imagen generalmente unilateral, uniloculada y econegativa, localizada en el lateral de hemiabdomen inferior debido a su pequeño tamaño, y no en pelvis fetal. Realizaremos siempre el diagnóstico diferencial con otras patologías intraabdominales como el hidrometocolpos, lesiones genitourinarias (megavejiga), o quistes del uraco, suprarrenales y gastrointestinales.

La vía de parto va a ser inicialmente vaginal, independiente de que se trate de un quiste simple o complicado (7). Algunos autores hablan incluso de partos con rotura del quiste durante el mismo sin que la rotura tuviera repercusión en el estado neonatal (3). La inducción del parto o la cesárea se propone en aquellos casos de sospecha de torsión ovárica u otras complicaciones derivadas del quiste, así como en aquellos casos en los que por el tamaño se sospeche la posibilidad de parto distócico $(3,8)$. En todos los casos presentados menores de $5 \mathrm{~cm}$, los partos fueron eutócicos con buenos resultados perinatales, lo que apoya la decisión de optar por esta vía en los quistes de pequeño tamaño.

En general, los quistes simples de menos de 5 $\mathrm{cm}$ tienden a resolverse espontáneamente en los primeros 4 a 6 meses de vida, y por lo tanto, no requieren tratamiento quirúrgico. La mayoría de los quistes de nuestro estudio, que fueron controlados ecográficamente cada 2 semanas durante el embarazo, disminuyeron su tamaño antes del parto o llegaron a resolverse espontáneamente tras el nacimiento. Esto apoya la recomendación que hasta ahora se hacía en todas las publicaciones previas de mantener una actitud expectante y conservadora.

En cuanto al tratamiento mediante punción ecoguiada intraútero, podemos decir que se trata de una práctica de dudosos resultados. Podría estar indicada en quistes de mayor tamaño $(>5 \mathrm{~cm})$ que tienen mayores probabilidades de dar lugar a complicaciones intraútero. Sin embargo, se ha observado un mayor riesgo de hemorragia, infección o incluso diseminación de tumores de estirpe maligna, siendo elevado el número de casos que recidivan, por lo que su práctica no estaría recomendada (7,9-11).

Debemos tener en cuenta que los quistes detectados en casi todos los casos eran quistes no complicados y unilaterales, que no llegaron a alcanzar gran tamaño en su evolución. Solamente el caso del quiste de $90 \mathrm{~mm}$ recibió tratamiento quirúrgico posnatal, por dificultad respiratoria debido al efecto masa que el tamaño del quiste ejercía a nivel torácico, sin que el parénquima pulmonar estuviera afectado; el resultado anatomopatológico fue de quiste folicular.

La cirugía posnatal se recomienda en los quistes simples mayores de $5 \mathrm{~cm}$ de diámetro, que tienen más riesgo de torsión ovárica y necrosis, y para quistes complejos, que frecuentemente ya están torsionados en el momento del diagnóstico (12-14).

Los quistes complejos pueden distinguirse en la ecografía posnatal (15) por la presencia de paredes hiperecogénicas, contornos irregulares, paredes espesas, o presencia de sedimento (Figura 2). En estos casos está indicada la exploración quirúrgica de forma electiva. La torsión y el infarto ovárico en periodo neonatal puede producir autoamputación del ovario, que se presenta como un quiste móvil en el abdomen, y que puede adherirse a las asas intestinales, produciendo obstrucción intestinal o complicarse con rotura del quiste y pérdida ovárica bilateral (16-18). En el caso de que exista clínica de obstrucción intestinal o abdomen agudo, situación poco frecuente, la cirugía deberá realizarse de manera urgente.

La vía quirúrgica clásica ha sido la laparotomía infraumbilical. Otra opción, cada vez más aceptada, es la vía laparoscópica, que permite un tratamiento mínimamente invasivo mediante punción y destechamiento del quistes en los casos en que se sospeche un origen hemorrágico o necrótico, que son la mayoría (Figura 3). En los raros casos en que la sospecha sea tumoral (diagnóstico infrecuente en neonatos, correspondiendo en su mayoría a teratomas maduros) la recomendación es realizar una disección del quiste respetando en lo posible el tejido ovárico (19-21). Algunos autores proponen la punción ecoguiada de los quistes (21), aunque como ya hemos comentado esta práctica no está libre de complicaciones (22). Un esquema de manejo para los quistes de ovario neonatales se puede observar en la (Figura 4) (23).

Podemos decir que los quistes ováricos de menor tamaño $(<5 \mathrm{~cm})$ tienen un mejor pronóstico y en la mayoría de los casos se resuelven espontáneamente o bien no aumentan su tamaño (24). Es por esto que recomendamos una actitud conservadora y seguimiento posnatal de aquellos fetos a los que se les detecta un quiste ovárico $<5 \mathrm{~cm}$, los cuales presentan un porcentaje de complicaciones antenatal mínimo, siendo además la vía vaginal la de elección para el parto. En su mayoría, estos quistes se resolverán por sí solos en un periodo aproximado de 6 meses. Sin embargo, aquellos quistes que superan esta medida límite de los $5 \mathrm{~cm}$ y no disminuyen de tamaño pueden precisar tratamiento 


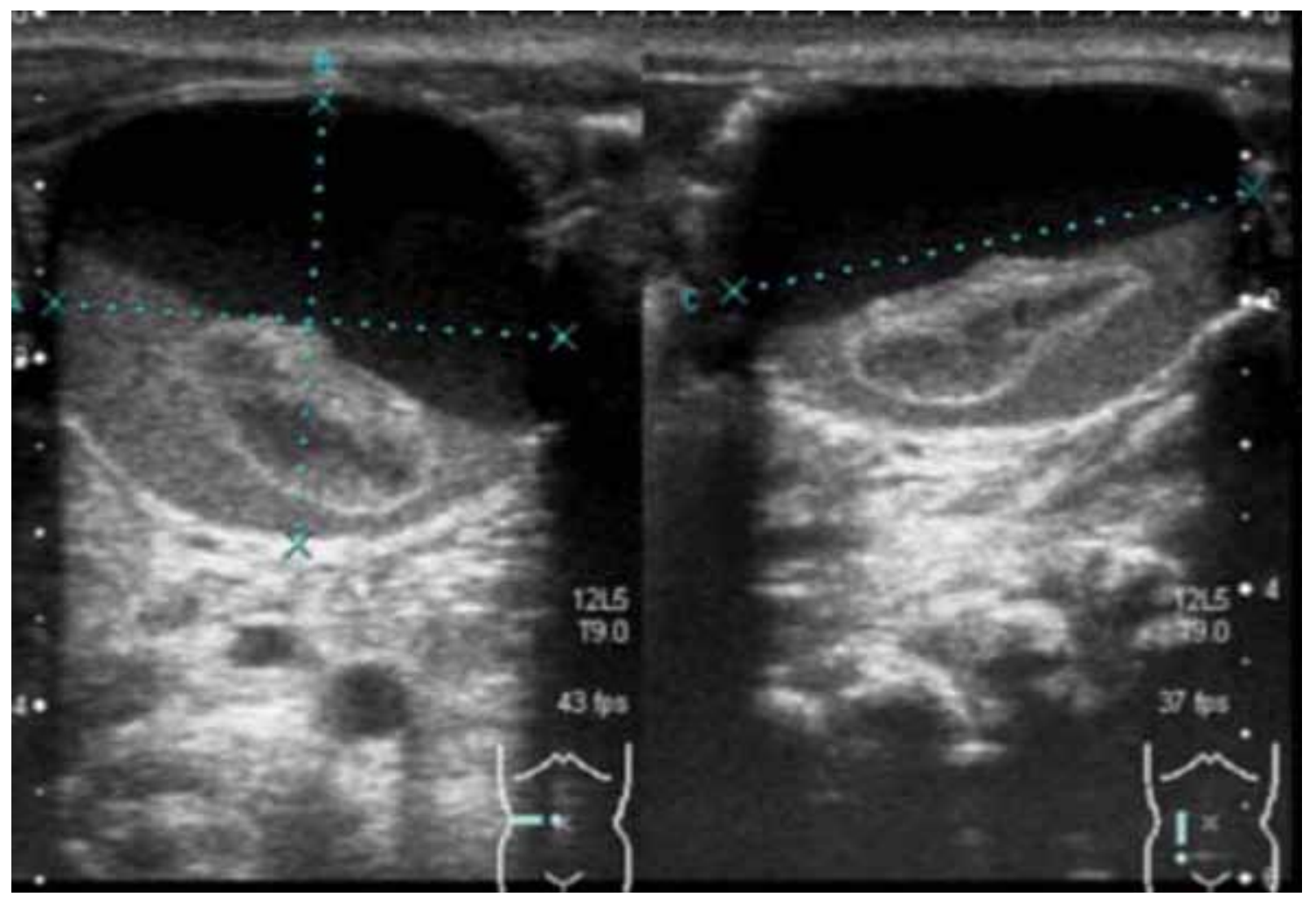

Figura 2. Aspecto ecográfico de quiste de ovario complicado posparto.

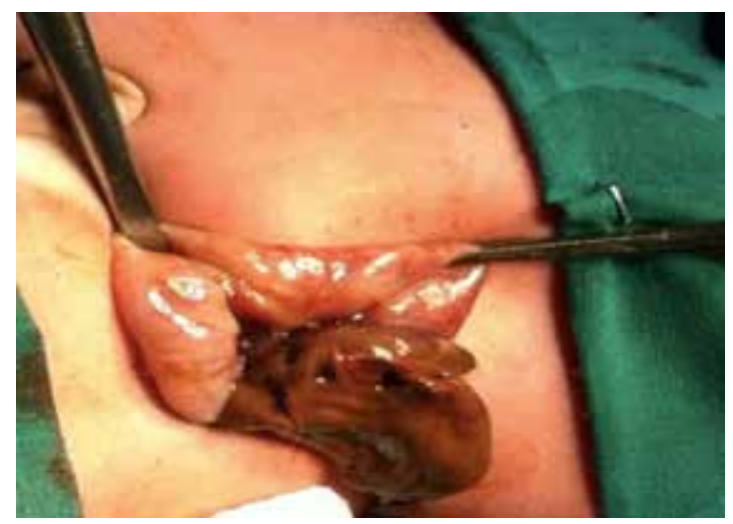

Figura 3. Quiste de ovario de origen hemorrágico, autoamputado y adherido a las asas intestinales. quirúrgico posparto, por lo que deberán realizarse controles prenatales frecuentes, pudiendo ser necesario incluso la finalización de la gestación en caso de quistes de gran tamaño o complicados.

\section{CONCLUSIÓN}

La ecografía del tercer trimestre es fundamental en el diagnóstico y seguimiento de los quistes ováricos fetales. Los quistes ováricos de menor tamaño $(<5 \mathrm{~cm})$, tienen buen pronóstico y la mayoría de los casos se resuelven espontáneamente o bien no aumentan su tamaño. Recomendamos una actitud conservadora y seguimiento posnatal de los fetos. Aquellos quistes que superan los $5 \mathrm{~cm}$ y no disminuyen de tamaño pueden precisar tratamiento quirúrgico posparto. 


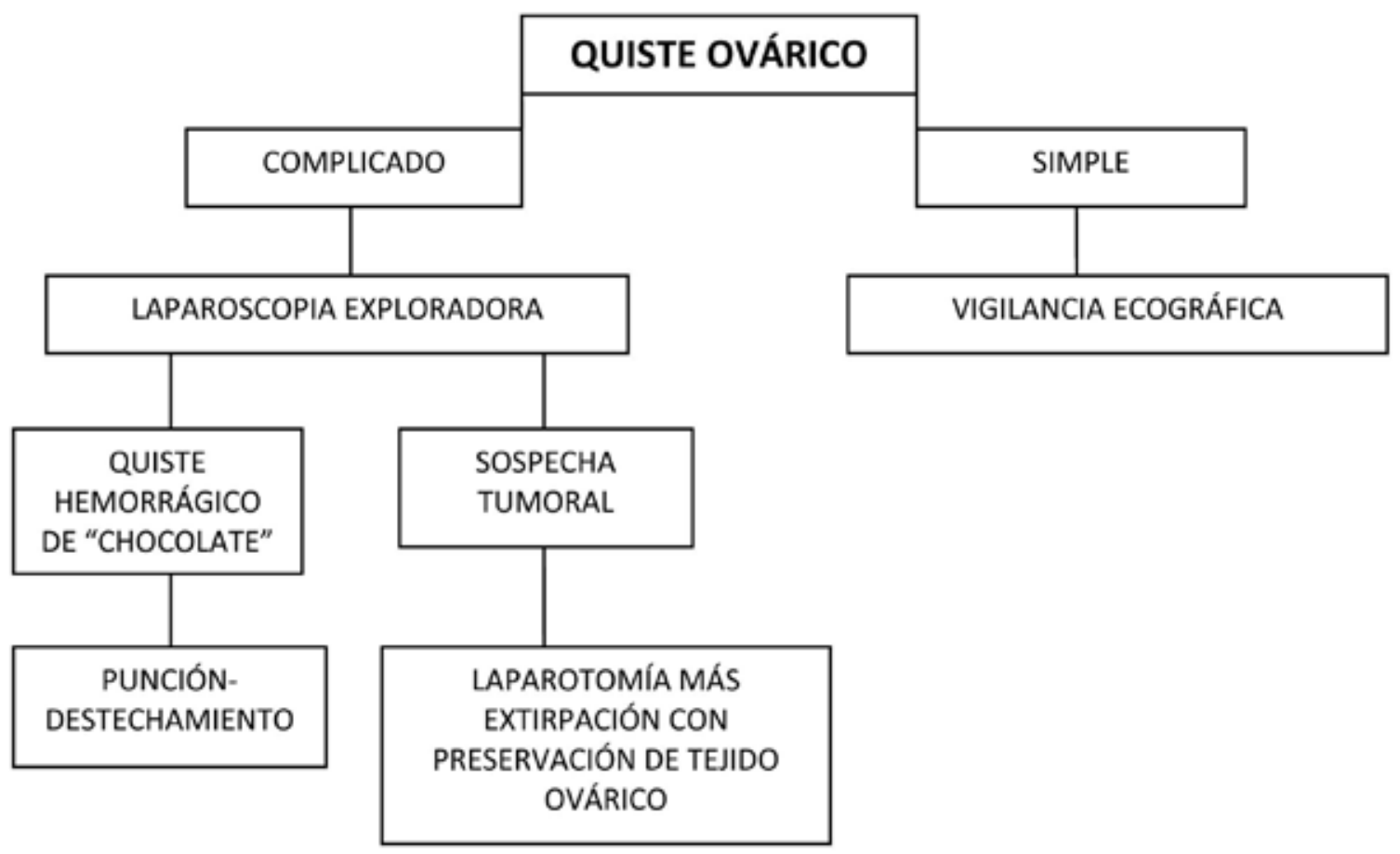

Figura 4. Esquema de manejo del quiste de ovario neonatal.

\section{REFERENCIAS}

1. Muñoz H, Wenzel C, Hasbun J, Neumann P, lleible S, Lazaretian $\mathrm{M}$, et al. Quiste ovárico fetal, diagnóstico antenatal y resultados perinatales. Rev Chil Obstet Ginecol 1998;63:310-4.

2. Pardo RA, Nazer J. Quiste ovárico fetal: diagnóstico ecográfico prenatal. Evolución y tratamiento postnatal. Casos clínicos. Rev Méd Chile 2003;131:665-8.

3. Gratacos E, Gómez R, Nikolaides K, Romero R, Cabero L. Quistes ováricos del feto. Medicina fetal. 1a edición. Buenos Aires-Madrid. Editorial Panamericana, 1991;459-63.

4. Godinho AB, Cardoso E, Melo MA, Gonçalves M, Da Graca LM. Ultrasonographic diagnosis of fetal ovarian cysts: five cases in five years. J Matern Fetal Neonatal Med 2008;21:875-9.

5. Dimitraki M, Koutlaki N, Nikas I, Mandratzi T, Gourovanidis $\mathrm{V}$, Kontomanolis $\mathrm{E}$, et al. Fetal ovarian cysts. Our clinical experience over 16 cases and review of the literature. J Matern Fetal Neonatal Med 2012;25:222-5.

6. Nemec U, Nemec SF, Bettelheim D, Brugger PC, Horcher E, Schöpf V, et al. Ovarian cysts on prenatal MRI. Eur J Radiol 2012;81:1937-44.

7. Heling KS, Chaovi R, Kirchmair F, Stadia S, Bollmann R. Fetal ovarian cysts: prenatal diagnosis, management and postnatal outcome. Ultrasound Obstet Gynecol 2002;20:47-50.
8. Rizzo N, Gabrielli S, Perolo A, Pilu G, Cacciari A, Domini $R$, et al. Prenatal diagnosis and management of fetal ovarian cysts. Prenat Diagn 1989;9:97-103.

9. Meizner L, Levy A, Katz M, Maresh AJ, Glezerman M. Fetal ovarian cysts: prenatal ultrasonographic detection, postnatal evaluation and treatment. Am J Obstet Gynecol 1991;164:874-8.

10. Heling KS, Chaovi R, Kirchmair F, Stadia S, Bollmann R. Prenatal diagnosis of congenital neuroblastoma. Analysis of 4 cases and review of the literature. Fetal Diagn Ther 1999;14:47-52.

11. Ben-Ami I, Kogan A, Fuchs N, Smorgick N, Mendelovic S, Lotan G, Herman A, Maymon R. Long-term follow-up of children with ovarian cysts diagnosed prenatally. Prenat Diagn 2010;30:342-7.

12. Słodki M, Janiak K, Szaflik K, Respondek-Liberska M. Fetal echocardiography before and after prenatal aspiration of a fetal ovarian cyst. Ginekol Pol 2009;80:629-31.

13. Monnery-Noché ME, Auber F, Jouannic JM, Bénifla $\mathrm{JL}$, Carbonne B, Dommergues M, et al. Fetal and neonatal ovarian cysts: is surgery indicated? Prenat Diagn 2008;28:15-20.

14. Brandt ML, Luks FI, Filiatrault D, Garel L, Desjardins $J G$, Youssef S. Surgical indications in antenatally diagnosed ovarian cysts. J Pediatr Surg 1991;26:276-82.

15. Noia G, Riccardi M, Visconti D, Pellegrino M, Quattrocchi $\mathrm{T}$, Tintoni $\mathrm{M}$, et al. Invasive fetal therapies: 
approach and results in treating fetal ovarian cysts. $\mathrm{J}$ Matern Fetal Neonatal Med 2011. J Clin Res Pediatr Endocrinol 2010;2:28-33.

16. Bagolan P, Rivosecchi M, Giorlandino C, Bilancioni E, Nahom A, Zaccara A, et al. Prenatal diagnosis and clinical outcome of ovarian cysts. J Pediatr Surg 1992;27:879-81.

17. Nussbaum AR, Sanders RC, Hartman DS, Dudgeon DL, Parmley TH. Neonatal ovarian cysts: Sonographicpathologic correlation. Radiology 1988;168:817-21.

18. Schenkman L, Weiner TM, Philips JD. Evolution of the surgical management of neonatal ovarian cysts: laparoscopic-assisted transumbilical extracorporeal ovarian cystectomy (LATEC). J Laparoendosc Adv Surg Tech 2008;18:635-40.

19. Lin JY, Lee Zf, Chang YT. Transumbilical management for neonatal ovarian cysts. J Pediatr Surg 2007;42:2136-9.
20. Marinković S, Jokić R, Bukarica S, Mikić AN, Vucković $\mathrm{N}$, Antić J. Surgical treatment of neonatal ovarian cysts. Med Pregl 2011;64:408-12.

21. Puligandla PS, Laberge JM. Lethal outcome after percutaneous aspiration of a presumed ovarian cyst in a neonate. Semin Pediatr Surg 2009;18:119-21.

22. Kessler A, Nagar H, Graif M. Percutaneous drainage as the treatment of choice for neonatal ovarian cysts. Pediatr Radiol 2006;36:954-8.

23. Shimada T, Miura K, Gotoh H, Nakayama D, Masuzaki $\mathrm{H}$. Management of prenatal ovarian cysts. Early Hum Dev 2008;84:417-20.

24. Słodki M, Janiak K, Szaflik K, Wilczyński J, Oszukowski P, Chilarski A, Respondek-Liberska M. Fetal echocardiography in fetal ovarian cysts. Ginekol Pol 2008;79:347-51. 